\title{
Clifford-Wolf translations of left invariant Randers metrics on compact Lie groups*
}

\author{
Shaoqiang Deng ${ }^{1}$ and Ming $\mathrm{Xu}^{2} \dagger$ \\ ${ }^{1}$ School of Mathematical Sciences and LPMC \\ Nankai University \\ Tianjin 300071, P. R. China \\ ${ }^{2}$ Department of Mathematical Sciences \\ Tsinghua University \\ Beijing 100084, P. R. China
}

\begin{abstract}
A Clifford-Wolf translation of a connected Finsler space is an isometry which moves each point the sam distance. A Finsler space $(M, F)$ is called Clifford-Wolf homogeneous if for any two point $x_{1}, x_{2} \in M$ there is a Clifford-Wolf translation $\rho$ such that $\rho\left(x_{1}\right)=x_{2}$. In this paper, we study Clifford-Wolf translations of left invariant Randers metrics on compact Lie groups. The mian result is that a left invariant Randers metric on a connected compact simple Lie group is CliffordWolf homogeneous if and only if the indicatrix of the metric is a round sphere with respect to a bi-invariant Riemannian metric. This presents a large number of examples of non-reversible Finsler metrics which are Clifford-Wolf homogeneous.

Mathematics Subject Classification (2000): 22E46, 53C30.

Key words: Finsler spaces, Clifford-Wolf translations, Killing vector fields, compact Lie groups, left invariant Randers metrics.
\end{abstract}

\section{Introduction}

Let $(M, g)$ be a homogeneous Riemannian manifold. An isometry of the Riemannian manifold $(M, g)$ is called a Clifford-Wolf translation (or simply CW-transformation) if it moves each point of $M$ the same distance. If for any two points $x_{1}, x_{2} \in M$, there is a CW-translation $\rho$ such that $\rho\left(x_{1}\right)=x_{2}$, then $(M, g)$ is called Clifford-Wolf homogeneous (or simply CW-homogeneous).

The general merit of the study of CW-translations lies in the fact that they are closely related to the study of homogeneous Riemannian manifolds. Let $\Gamma$ be a properly discontinuous subgroup of the full group of isometries of $(M, g)$, which acts freely on $M$. It is a very important problem in Riemannina geometry to find out the conditions that the quotient manifold $M / \Gamma$ is again a homogeneous Riemannian manifolds. Through the work of a number of researchers, we can say that at least in the cases that $(M, g)$

\footnotetext{
*Supported by NSFC(no.10671096 and 10971104) and SRFDP of China.

${ }^{\dagger}$ Corresponding author. E-mail: mgxu@math.tsinghua.edu.cn
} 
is a Riemannian symmetric space, or a homogeneous Riemannian manifold of nonpositive curvature, or a homogeneous Riemannian manifold admitting a transitive group of isometries which is semisimple of the noncompact type, $M / \Gamma$ is a homogeneous Riemannian manifold, provided that $\Gamma$ consists of $\mathrm{Cw}$-translations. Moreover, in the first two cases it is proved that the above condition is also sufficient; see [WO60, WO62, WO64 and DMW86 for the details.

In view of the the above results, it is an important problem to determine all the CW-transformations of a given Riemannian manifold, particularly for the homogeneous ones. For Riemannian symmetric spaces this problem was first completely settled by J. A. Wolf in [WO62]; see also [FR63, OZ69, OZ74] for other proofs of the results. The related results has important application in the study of homogeneous Riemannian manifolds of negative curvature; see for example [HE74, AW76].

Recently the study on CW-translation has become active. In BN08-1, BN08-2, BN09, Berestovskii and Nikonorov studied the local one-parameter group of CWtranslations of general Riemannian manifolds. The main result is that there is a correspondence between local one-parameter groups of CW-translations and Killing vector fields of constant length. They also obtained a classification of connected simply connected CW-homogeneous Riemannian manifolds. The list consists of the euclidean spaces, the odd-dimensional spheres with constant curvature, connected simply connected compact simple Lie groups with bi-invariant Riemannian metrics and the direct products of the above manifolds. Notice that there are also a local version of the related notion of CW-homogeneous Riemannian manifolds; see the above cited papers above.

In our recent papers [DE12] and [DM12, we initiated the study of CW-translations of Finsler spaces. The notions of CW-translations and CW-homogeneity have been generalized to the Finslerian setting. It is proved that the correspondence between the local one-parameter subgroup of CW-translations and the Killing vector fields of constant length is also valid for a Finsler space; see the next section for the details. We also studied this problem for a special type of Finsler spaces-Randers spaces and obtained a necessary and sufficient condition for a smooth vector field $X$ on a homogeneous Randers space $(G / H, F)$, with $H$ connected, to be a Killing vector field of constant length. This result gives a complete classification for all the local one-parameter subgroup of CW-translations of a homogeneous Randers space.

In this paper we continue our study on this topic. Our main idea is to study the condition for a homogeneous Randers space to be CW-homogeneous, and futhermore, to classify all the CW-homogeneous Randers spaces. However, due to the complexity of the problem, we will only deal with left invariant Randers metrics on a compact simple Lie group. The main result of this paper is the following

Theorem 1.1 Let $G$ be a compact connected simple Lie group and $F$ be a left invariant Randers metric. Then $(G, F)$ is $C W$-homogeneous if and only if the indicatrix of $F$ in $\mathfrak{g}$ is a round sphere with respect to a bi-invariant metric.

Recall that the indicatrix of a Finsler space $(M, F)$ at a point $x \in M$ is defined to be

$$
\mathcal{I}_{x}=\left\{y \in T_{x}(M) \mid F(x, y)=1\right\} .
$$

Notice that in the above theorem, we have identified $\mathfrak{g}$ with $T_{e} G$. On the other hand, since $G$ is simple, up to a positive scalar the bi-invariant Riemannian metric on $G$ is 
unique. Therefore the statement of the theorem have no independence on the specific bi-invariant metric. The above theorem says that a left invariant Randers metric on a compact simple is CW-homogeneous if and only if it solves the Zermelo's navigation problem of a bi-invariant Riemannian metric under the influence of certain external vector field. Put another way, this theorem presents many examples of non-reversible Finsler spaces which are CW-homogeneous. It would be an interesting problem to classify CW-homogeneous Finsler spaces, either reversible or non-reversible.

The arrangement of this paper is as the following. In Section 2, we recall some notions and known results on Finsler geometry and CW-translations of Finsler spaces. In Section 3, we study Killing vector fields of left invariant Randers metrics and obtain a complete description of those with constant length. In Section 4, we first state a theorem (Theorem 4.1) and then use this theorem to give a proof of Theorem 1.1. Finally, in Section 5, we give a case by case proof of Theorem 4.1 .

\section{Preliminary}

Finsler geometry is introduced by Riemann in his celebrated lecture on the foundations of geometry, addressed in 1854. Due to the complexity of the problem, the study of Finsler geometry was dormant for a rather long period. In 1918, Finsler studied the variation problem of Finsler spaces in his doctoral dissertation, which initiated the systematic study of Finsler geometry. For fundamental properties of Finsler spaces, we refer the readers to [BCS00, [CS05] and [SH01.

Definition 2.1 A Finsler metric on a manifold $M$ is a continuous function $F: T M \rightarrow$ $\mathbb{R}^{+}$, which is smooth on the slit tangent bundle $T M \backslash 0$. In any local standard coordinates $\left(x^{i}, y^{j}\right)$ for $T M, F$ satisfies the following conditions:

(1) $F(x, y)>0$ for any $y \neq 0$.

(2) $F(x, \lambda y)=\lambda F(x, y)$ for any $y \in T M_{x}$, and $\lambda>0$.

(3) The Hessian matrix defined by $g_{i j}=\frac{1}{2}\left[F^{2}\right]_{y^{i} y^{j}}$ is positive definite.

An important example is a Finsler metric with the form $F=\alpha+\beta$, where $\alpha$ is a Riemannian metric and $\beta$ is a 1-form. This kind of Finsler metrics are called Randers metrics. Notice that the norm of the 1 -form $\beta$ with respect to the metric $\alpha$ must be smaller than 1 and they reduce to Riemannian metrics if and only if the $\beta$-terms vanish. Randers metrics are among the most important examples of non-Riemannian Finsler metrics in the study of geometry and physics.

Using the integration of $F$ along a path, we can define the arc length of piece-wise smooth curves. The "distance" of two points is then defined to be the infimum of the arc length of all the piece-wise smooth curves connecting them. We call it the distance function and denote as $d(\cdot, \cdot)$. In general, the distance function of a Finsler space is not reversible (i.e., $d(x, y)=d(y, x)$ for any $x, y)$ unless $F(x, y)=F(x,-y)$, for all $x \in M$ and $y \in T M_{x}$.

The notion of CW-translations can be generalized to Finsler geometry; see [DM12].

Definition 2.2 A $C W$-translation $\rho$ of a Finsler manifold $(M, F)$ is an isometry of $(M, F)$ such that $d(x, \rho(x))$ is a constant function. 
In the Riemannian case, the interrelation between CW-translations and Killing vector fields of constant lengths is studied by V.N.Berestovskii and Yu.G.Nikonorov. The key observation is that the flow curves of a Killing vector field of constant length are geodesics, so Killing vector fields of constant length can be used to generate oneparameter groups of CW-tranlations when the parameter var1able is close to 0 . This observation is still valid in the Finslerian case, except that the Killing vector fields of constant lengths can only generate CW-translations with positive small parameter variables when $F$ is not absolutely homogeneous. In fact, we have the following interrelation theorems ([DM12]).

Theorem 2.3 Let $(M, F)$ be a complete Finsler manifold with a positive injective radius. If $X$ is a Killing vector field of constant length, then the flow $\phi_{t}$ generated by $X$ is a $C W$-translation for all sufficiently small $t>0$.

Theorem 2.4 Let $(M, F)$ be a compact Finsler manifold. Then there is a $\delta>0$, such that any $C W$-translation $\rho$ with $d(x, \rho(x))<\delta$ is generated by a Killing vector field of constant length.

We have also generalized the concept of (restrictively) CW-homogeneous space in Finsler geometry.

Definition 2.5 A Finsler manifold $(M, F)$ is called $C W$-homogeneous if for any two points $x_{1}, x_{2} \in M$, there is a $C W$-translation $\rho$ such that $\rho\left(x_{1}\right)=x_{2}$. It is called restrictively $C W$-homogeneous if for any point $x \in M$, there is a neighborhood $V$ of $x$, such that for any two points $x_{1}, x_{2} \in V$ there is a $C W$-translation $\rho$ mapping $x_{1}$ to $x_{2}$.

Notice that the set of CW-translations of a Finsler space is generally not a subgroup of the full group of isometries. Therefore, restrictive CW-homogeneity is a weaker notion than $\mathrm{CW}$-homogeneity. In the case that $(M, F)$ is a compact Finsler manifold, the interrelation between $\mathrm{CW}$-translations and Killing vector fields of constant length gives an equivalent description of the restrictive CW-homogeneity, namely, the Finsler space $(M, F)$ is restrictively $\mathrm{CW}$-homogeneous if and only if any non-vanishing tangent vector can be extended to a Killing vector field of constant length.

\section{Left invariant Randers metrics and Killing vector fields of constant length}

Let $G$ be a connect compact Lie group with Lie algebra $\mathfrak{g}$. A Finsler metric $F$ on $G$ is called left invariant if the left translation group $L(G)$ is contained in the isometry group of $F$. A left invariant Finsler metric is completely determined by its restriction to $T_{e} G \cong \mathfrak{g}$. A left invariant Randers $F=\alpha+\beta$ is then determined by an inner product on $\mathfrak{g}$ and an element of $\mathfrak{g}^{*}$, which define the $\alpha$ and $\beta$ terms respectively. Let $\langle$,$\rangle be the$ inner product induced by $\alpha$ and $\langle,\rangle_{\text {bi }}$ be a fixed $\operatorname{Ad}(G)$-invariant inner product on $\mathfrak{g}$ induced by a bi-invariant metric on $G$, then the restriction of $F$ to $T_{e} G$ can be written as $F(y)=\sqrt{\langle y, y\rangle}+\langle y, V\rangle_{\text {bi }}$ for some $V \in \mathfrak{g}$.

The Killing vector fields with constant length of a left invariant Randers metric is the key subject for our considerations. In [DM12, we have already discussed some 
techniques for determining Killing vector fields $X \in \mathfrak{g}$ with constant length, and found some examples in which the $\mathrm{CW}$-translations are different from those Riemannian ones. Any Killing vector field $X$ belongs to the Lie algebra of the full isometry group of $F$, which contains $\mathfrak{g}$ as a Lie subalgebra corresponding to the Lie subgroup $L(G)$. Therefore it is important to compute the full isometries of $(G, F)$. In general, it is rather difficult to get a complete classification of all the isometry groups of left invariant Randers metrics on a compact Lie group. However, the following lemma tells us that its identity component is contained in $L(G) R(G)$ when $G$ is simple (i.e., $\mathfrak{g}$ is simple).

Lemma 3.1 Let $G$ be a compact connected simple Lie group and $F$ a left invariant Randers metric on $G$. Then the identity component $I_{0}(G, F)$ of the full group $I(M, F)$ of isometries of $(G, F)$ is contained in $L(G) R(G)$.

Proof. Let $F=\alpha+\beta$ be the standard decomposition for the left invariant Randers metric. Then we have $L(G) \subset I(G, F) \subset I(G, \alpha)$, i.e., $\alpha$ is a left invariant Riemannian metric. Since $G$ is compact connected simple, $I_{0}(G, \alpha)$ is contained in $L(G) R(G)$ ([OT76]). Thus $I_{0}(G, F) \subset I_{0}(G, \alpha)$ is also contained in $L(G) R(G)$.

Let $G^{\prime}$ be the subgroup of $G$ such that $R\left(G^{\prime}\right)$ is the connected component of the group of all isometric right translations, and denote $\operatorname{Lie}\left(G^{\prime}\right)=\mathfrak{g}^{\prime}$. Then obviously $I_{0}(G, F)=L(G) R\left(G^{\prime}\right)$. In the Lie algebra level, the Lie algebra of $I_{0}(G, F)$, i.e., the space of all Killing vector fields, is the direct sum of $\mathfrak{g}$ and $\mathfrak{g}^{\prime}$.

The following theorem gives a criterion for a Killing vector field $\left(X, X^{\prime}\right) \in \mathfrak{g} \oplus \mathfrak{g}^{\prime}$ of the left invariant Randers metric $F$ to have constant length.

Theorem 3.2 If $\left(X, X^{\prime}\right) \in \mathfrak{g} \oplus \mathfrak{g}^{\prime}$ generates a Killing vector field of constant length of a left invariant Randers metric $F$, then either $X=0$ or $X^{\prime}$ belongs to the center $\mathfrak{c}\left(\mathfrak{g}^{\prime}\right)$ of $\mathfrak{g}^{\prime}$.

Proof. Let $F(y)=\sqrt{\langle y, y\rangle}+\langle y, V\rangle_{\mathrm{bi}}$ be the restriction of $F$ to $T_{e} G$. If $\left(X, X^{\prime}\right) \in \mathfrak{g} \oplus \mathfrak{g}^{\prime}$ generates a Killing vector field of constant length for $F$, then the projection of the $\operatorname{Ad}\left(L(G) R\left(G^{\prime}\right)\right)$-orbit of $\left(X, X^{\prime}\right)$ in $\mathfrak{g}$ has the same $F$ values, i.e.,

$$
\begin{aligned}
F\left(\operatorname{Ad}\left(\left(g, g^{\prime}\right)\right)\left(X, X^{\prime}\right)\right) & =\alpha\left(\operatorname{Ad}(g) X-\operatorname{Ad}\left(g^{\prime}\right) X^{\prime}\right)+\left\langle\operatorname{Ad}(g) X-\operatorname{Ad}\left(g^{\prime}\right) X^{\prime}, V\right\rangle_{\mathrm{bi}} \\
& \equiv \text { constant, } \forall g \in G, g^{\prime} \in G^{\prime}
\end{aligned}
$$

Since $\left\langle\operatorname{Ad}\left(g^{\prime}\right) X^{\prime}, V\right\rangle_{\mathrm{bi}}=\left\langle X^{\prime}, V\right\rangle$, we see that for any fixed $g \in G, \alpha\left(\operatorname{Ad}(g) X-\operatorname{Ad}\left(g^{\prime}\right) X^{\prime}\right)$ is a constant function of $g^{\prime} \in G^{\prime}$. So

$$
\begin{aligned}
\left\langle\operatorname{Ad}(g) X, \operatorname{Ad}\left(g^{\prime}\right) X^{\prime}\right\rangle_{\alpha} & =\frac{1}{2}\left(\alpha(\operatorname{Ad}(g) X)+\alpha\left(\operatorname{Ad}\left(g^{\prime}\right) X^{\prime}\right)-\alpha\left(\operatorname{Ad}(g) X-\operatorname{Ad}\left(g^{\prime}\right) X^{\prime}\right)\right) \\
& =\frac{1}{2}\left(\alpha(\operatorname{Ad}(g) X)+\alpha\left(X^{\prime}\right)-\alpha\left(\operatorname{Ad}(g) X-\operatorname{Ad}\left(g^{\prime}\right) X^{\prime}\right)\right)
\end{aligned}
$$

is a constant function of $g^{\prime} \in G^{\prime}$, for any fixed $g \in G$. Now Select

$$
g^{\prime}=\exp \left(t_{1} Y_{1}\right) \cdots \exp \left(t_{n} Y_{n}\right)
$$

Taking the derivative with respect to all the $t_{i}$ 's and evaluating at 0 , we easily deduce that for any $g \in G$, the vector $\operatorname{Ad}(g) X$ is orthogonal to the ideal generated by $\left[X^{\prime}, \mathfrak{g}^{\prime}\right]$ 
in $\mathfrak{g}^{\prime}$ with respect to $\alpha$. Since the above assertion holds for any $g \in G$, we see that the ideal generated by $X$ in $\mathfrak{g}$ is orthogonal to the ideal generated by $\left[X^{\prime}, \mathfrak{g}^{\prime}\right]$ in $\mathfrak{g}^{\prime}$. Since $\mathfrak{g}$ is simple, this implies that either $X=0$ generates the 0 ideal, or $X \neq 0$ and it generates the ideal $\mathfrak{g}$. Notice that in the later case we have $\left[X^{\prime}, \mathfrak{g}^{\prime}\right]=0$, i.e., $X^{\prime} \in \mathfrak{c}\left(\mathfrak{g}^{\prime}\right)$. This completes the proof of the theorem.

Using a similar argument we can prove the following modification of Theorem 3.2 , which allows more general $G^{\prime}$.

Theorem 3.3 Let $G$ be a compact connected simple Lie group, and $G^{\prime}$ a closed connected subgroup of $G$ with Lie algebra $\mathfrak{g}^{\prime}$. Let $F$ be a $L(G) R\left(G^{\prime}\right)$-invariant Randers metric on $G$. If $\left(X, X^{\prime}\right) \in \mathfrak{g} \oplus \mathfrak{g}^{\prime}$ generates a Killing vector field of constant length, then either $X=0$ or $X^{\prime}$ belongs to the center $\mathfrak{c}\left(\mathfrak{g}^{\prime}\right)$ of $\mathfrak{g}^{\prime}$.

In Theorem 3.3, we treat $(G, F)$ as the homogeneous Randers space, in which is viewed as the coset space $G=\left(L(G) R\left(G^{\prime}\right)\right) / H$ and $H$ can be identified with the quotient of $G^{\prime}$ by a discrete normal subgroup. The Lie algebra $\mathfrak{h}$ of $H$ is isomorphic to $\mathfrak{g}^{\prime}$ and it is a "diagonal" in $\mathfrak{g} \oplus \mathfrak{g}^{\prime}$. The projection from $\left(X, X^{\prime}\right) \in \mathfrak{g} \oplus \mathfrak{g}^{\prime}$ to $\mathfrak{m} \cong \mathfrak{g}$ is just $X-X^{\prime}$.

For simplicity, we assume that $\left(X, X^{\prime}\right) \in \mathfrak{g} \oplus \mathfrak{c}\left(\mathfrak{g}^{\prime}\right)$, with $X \neq 0$, generates a Killing vector field of constant length 1 for the $L(G) R\left(G^{\prime}\right)$-invariant Randers metric $F$ on $G$. Then the projection from the orbit to $\mathfrak{g}$ is just a shift by $-X^{\prime}$. Its projection in $\mathfrak{g}$ is contained in an indicatrix ellipsoid $S$ for $F$ if and only if the $\operatorname{Ad}(G)$-orbit of $X$ is contained in another ellipsoid $S^{\prime}$, which is the indicatrix for another left invariant Randers metric $F^{\prime}$. Then $X \in \mathfrak{g}$ generates a Killing vector field of constant length 1 of $F^{\prime}$, and $X^{\prime}$ must be inside the indicatrix ellipsoid of $F^{\prime}$. The Randers metric $F$ is invariant under the right multiplications in $G^{\prime}$ if and only its indicatrix $S$ in $\mathfrak{g}$ is $\operatorname{Ad}\left(G^{\prime}\right)$-invariant. By Theorem 3.3 , the shifting from $S$ to $S^{\prime}$ by $X^{\prime}$ is $\operatorname{Ad}\left(G^{\prime}\right)$-invariant, so $F^{\prime}$ is also $L(G) R\left(G^{\prime}\right)$-invariant. The correspondence from $F^{\prime}$ to $F$ is similar. We have thus proved the following theorem.

Theorem 3.4 Let $G$ be a compact Lie group with simple Lie algebra $\mathfrak{g}, G^{\prime}$ a closed connected subgroup of $G$ with Lie algebra $\mathfrak{g}^{\prime}$. Then for any $\left(X, X^{\prime}\right) \in \mathfrak{g} \oplus \mathfrak{c}\left(\mathfrak{g}^{\prime}\right), X \neq 0$, and $l>0$, there is an one-to-one correspondence between the following two sets:

(1) The set of all $L(G) R\left(G^{\prime}\right)$-invariant metrics such that $\left(X, X^{\prime}\right)$ generates a Killing vector field of constant length $l$.

(2) The set of all $L(G) R\left(G^{\prime}\right)$-invariant metrics such that $X$ generates a Killing vector field of constant length $l$ and the length of $X^{\prime}$ with respect to the Randers metric is less than $l$.

Theorem 3.4 provides a theoretical machinery to find left invariant Randers metrics with von-vanishing Killing vector fields of constant length. First we fix a nonzero $X \in \mathfrak{g}$ and find the left invariant Randers metrics such that $X$ generates a Killing vector field of constant length for this metric (as we did in the previous work DM12]). If its isometry group is the product of $L(G) R\left(G^{\prime}\right)$ as given above, then we can freely choose any $X^{\prime}$ from $\mathfrak{c}\left(\mathfrak{g}^{\prime}\right)$ which is shorter than $X$. In this way we get the a Randers metric by 
requiring its indicatrix to be the parallel shifting of the former one by $-X^{\prime}$. Then it is easily seen that the above Randers metric has a Killing vector field of constant length generated by $\left(X, X^{\prime}\right)$.

Besides the Killing vector fields generated by $\left(X, X^{\prime}\right) \in \mathfrak{g} \oplus \mathfrak{c}(\mathfrak{g})$ with $X \neq 0$, there are Killing vector fields of constant lengths generated by elements of the form $\left(0, X^{\prime}\right)$. It is easy to see that in this case $X^{\prime}$ can be any vector in $\mathfrak{g}^{\prime}$. Since the subgroup $R\left(G^{\prime}\right) \in I(G, F)$ commute with $L(G)$, and $L(G)$ acts transitively on $G, R\left(G^{\prime}\right)$ gives a group of $\mathrm{CW}$-translations which is neither new nor interesting for our consideration (see [DM12]).

\section{Left invariant CW-homogeneous Randers metrics on simple compact Lie groups}

We now apply Theorem 3.4 to give a proof of Theorem 1.1. The proof contains two steps. We first prove the statement of the theorem with $\mathrm{CW}$-homogeneity replaced by restrictive CW-homogeneity. Then we prove that, for a left invariant Randers metric on a connected simply connected compact simple Lie group, the CW-homogeneity and restrictive homogeneity are equivalent.

The first step of the proof needs the following theorem, which will be proved in the next section.

Theorem 4.1 Let $G$ be a compact connected simple Lie group with Lie algebra $\mathfrak{g}$. Then a generic $X \in \mathfrak{g}$ can not generate a Killing vector field of constant length when the left invariant Randers metric $F$ on $G$ is not Riemannian. The complement of all those generic elements is a subvariety with a codimension at least $\mathrm{rk}(\mathfrak{g})+1$.

We remark here the generic condition is some condition for the eigenvalue multiplicities when $\mathfrak{g}$ is realized as a Lie algebra of matrices, and the exact sense may depend on the explicit Lie algebras; see the interruption in Section 5 for the Lie algebras of $A_{n}, \geq 1, D_{n}$ with $n$ odd and $>2$, and $E_{6}$. Hence apparently the generic condition has not been precisely defined. However, what is important in the above theorem is the assertion on the codimension of the set of generic elements. In fact, in the proof of Theorem 4.1, we will only use the generic condition in the above mentioned three cases, in which the condition can be precisely described.

Proof of Theorem 1.1. Let $I_{0}(G, F)$ be the product $L(G) R\left(G^{\prime}\right)$, with $G^{\prime} \subset G$ and $R\left(G^{\prime}\right)$ the connected subgroup of all right translation isometries. Denote the Lie algebra of $I_{0}(G, F)$ by $\mathfrak{g} \oplus \mathfrak{g}^{\prime}$. If the indicatrix of the $L(G) R\left(G^{\prime}\right)$-invariant metric $F$ in $T_{e} G \cong \mathfrak{g}$ is a round sphere, centered at $-X^{\prime}$, with radius $r>0$ with respect to the bi-invariant metric, then $X^{\prime}$ is $\operatorname{Ad}\left(G^{\prime}\right)$-invariant, i.e., $X^{\prime}$ lies in $\mathfrak{c}(\mathfrak{g})$. Since the indicatrix of $F$ contains 0 , the length of $X^{\prime}$ with respect to the bi-invariant metric satisfies $\left|X^{\prime}\right|_{\mathrm{bi}}<r$. Any vector $\left(X, X^{\prime}\right)$, with $|X|_{b i}=r$, generates a Killing vector field of constant length, and these vectors exhaust all tangent directions in $T_{e} G$. Through left translations, the Killing vector fields of constant lengths can exhaust all tangent directions at any point. Thus $(G, F)$ is restrictively $\mathrm{CW}$-homogeneous.

Conversely, if $(G, F)$ is restrictively $\mathrm{CW}$-homogeneous, then any tangent vector $X^{\prime \prime} \in \mathfrak{g} \cong T_{e} G$, of length 1 with respect to $F$ can be extended to a Killing vector field 
of constant length 1 . Such a Killing vector field $\left(X, X^{\prime}\right)$ has either of the following two forms:

(1) $X \in \mathfrak{g}, X^{\prime} \in \mathfrak{c}\left(\mathfrak{g}^{\prime}\right)$, and $X=X^{\prime}+X^{\prime \prime}$;

(2) $\left(0, X^{\prime}\right)$, with $X^{\prime}=-X^{\prime \prime} \in \mathfrak{g}^{\prime}$.

Notice that if $F$ is Riemannian, then it must be a bi-invariant metric ([DM12]). Now suppose $F$ is not Riemannian and write $F$ as $F(y)=\sqrt{\langle y, y\rangle}+\langle y, V\rangle_{\text {bi }}$, where $y \in T_{e} G$ and $V$ is a non-vanishing vector in $\mathfrak{g}$. Then $\left[X^{\prime}, V\right]=\left[X^{\prime \prime}, V\right]=0$ for a Killing vector field $\left(X^{\prime}, X^{\prime \prime}\right)$ of constant length with the second form. In this case $X^{\prime \prime}$ is contained in a subspace with a lower dimension. So when $X^{\prime \prime}$ is generic, the corresponding Killing vector field $\left(X, X^{\prime}\right)$ is of the first form, i.e., $X^{\prime} \in \mathfrak{c}\left(\mathfrak{g}^{\prime}\right)$. Theorem 3.4 implies that $X$ generates a Killing vector field of constant length for another $L(G) R\left(G^{\prime}\right)$-invariant Randers metric $F^{\prime}$, whose indicatrix is just a shift of that of $F$. Notice that all the possible $X^{\prime}$ 's are contained in $\mathfrak{c}\left(\mathfrak{g}^{\prime}\right)$ which has a dimension at most $\operatorname{rk}(\mathfrak{g})$. For generic $X^{\prime \prime}$, the corresponding $X=X^{\prime}+X^{\prime \prime}$ must be generic in the sense of Theorem 4.1, otherwise $X$ belongs to a subvariety with a codimension at least $r k(\mathfrak{g})+1$, and this implies that $X^{\prime \prime}=X-X^{\prime}$ belongs to subvariety with a codimension at least 1 , which conflict with the assumption that $X^{\prime \prime}$ is generic. So the corresponding $F^{\prime}$ for $X$ must be Riemannian, or equivalently, its indicatrix must be the only shifting of that of $F$ with its center shifted back to 0 . Thus for a generic vector $X^{\prime \prime}$ with length 1 for $F$, the corresponding Killing vector field $\left(X, X^{\prime}\right)$ of constant length has the same $X^{\prime}$ factor, and the corresponding $F^{\prime}$ for the $X$ term is also the same. As the set of the Killing vector fields with a fixed constant length is a closed subset of the set of all the Killing vector fields, the above assertion is true for all $X^{\prime \prime}$ 's with length 1 for $F$. So $F^{\prime}$ is a restrictively $\mathrm{CW}$-homogeneous Riemannian metric, i.e., it is a bi-invariant Riemannian metric. Consequently the indicatrix of $F^{\prime}$ in $\mathfrak{g}$ is a round sphere. Hence its shifting, the indicatrix of $F$, is also a round sphere.

Up to now we have completed the first step of the proof. For the second step we only need to prove that for a left invariant Randers metric on a connected simply connected simple Lie group, the restrictive CW-homogeneity implies the CW-homogeneity.

By suitable scalar changes, we can assume that $\left\{\left.(X, V)|| X\right|_{\mathrm{bi}}=1\right\}$ generates all the Killing vector fields of constant length 1 with respect to $F$, in which the fixed $V$ satisfies $|V|_{\text {bi }}<1$. Then after a constant re-scaling of the parameter, any geodesic of $(G, F)$ starting from $g$ can be written as $\exp (t X) g \exp (-t V)$ with $t \geq 0$, for some $X \in \mathfrak{g}$ with $|X|_{\mathrm{bi}}=1$. The geodesic $\exp (t X) g \exp (-t V), t \in\left[0, t_{0}\right]$ from $g$ to $g^{\prime}$ is not minimizing if and only if there is another geodesic from $g$ to $g^{\prime}$ with the form $\exp \left(t X^{\prime}\right) g \exp (-t V)$, $\left|X^{\prime}\right|_{\mathrm{bi}}=1$ and $t \in\left[0, t^{\prime}\right]$ with $t^{\prime}<t_{0}$. This implies that

$$
\exp \left(t_{0} X\right) g \exp \left(-t_{0} V\right)=\exp \left(t^{\prime} X^{\prime}\right) g \exp \left(-t^{\prime} V\right)
$$

i.e., $\exp \left(t_{0} X\right)=\exp \left(t^{\prime} X^{\prime}\right) \exp \left(\left(t_{0}-t^{\prime}\right) \operatorname{Ad}(g) V\right)$. For the bi-invariant metric, the geodesic $\exp (t X), t \in\left[0, t_{0}\right]$ from the unit element $e$ to $g^{\prime} g^{-1}$ has a length $t_{0}$. The right side of (4.3) gives a path from $e$ to $\exp \left(t^{\prime} X^{\prime}\right) \exp \left(\left(t_{0}-t^{\prime}\right) \operatorname{Ad}(g) V\right)$, which is a geodesic from $e$ to $\exp \left(\left(t_{0}-t^{\prime}\right) V\right)$ with length $\left(t_{0}-t^{\prime}\right)|V|_{b i}$, and a geodesic from $\exp \left(\left(t_{0}-t^{\prime}\right) V\right)$ to $\exp \left(t^{\prime} X^{\prime}\right) \exp \left(\left(t_{0}-t^{\prime}\right) \operatorname{Ad}(g) V\right)$ with length $t^{\prime}$. The total length is less than $t_{0}$, so the geodesic $\exp (t X), t \in\left[0, t_{0}\right]$ is not minimizing with respect to the bi-invariant metric. The next lemma shows the converse statement is also true. 
Lemma 4.2 Let $X$ be a unit vector and $\exp (t X), t \in\left[0, t_{0}\right]$, a geodesic from e to $g=\exp \left(t_{0} X\right)$ which is not minimizing with respect to the bi-invariant metric. Then for any $V \in \mathfrak{g}$ with $|V|_{b i}<1$, there is $t^{\prime} \in\left[0, t_{0}\right)$ and a unit vector $X^{\prime} \in \mathfrak{g}$, such that

$$
g=\exp \left(t_{0} X\right)=\exp \left(t^{\prime} X^{\prime}\right) \exp \left(\left(t_{0}-t^{\prime}\right) V\right) .
$$

Proof. We construct a sequence $t_{n} \in\left[0, t_{0}\right]$, and a sequence of unit vectors $X_{n} \in \mathfrak{g}$ with respect to the bi-invariant metric, inductively as follows. Let $t_{1}$ and $X_{1}$ be the pair such that the geodesic $\exp \left(t X_{1}\right), t \in\left[0, t_{1}\right]$, is minimizing from $e$ to $g$. Suppose we have defined $t_{i}$ and $X_{i}$. Then we choose $t_{i+1}$ and $X_{i+1}$ to be the pair such that the geodesic $\exp \left(t X_{i+1}\right), t \in\left[0, t_{i+1}\right]$, is the shortest from $e$ to $g \exp \left(\left(t_{i}-t_{0}\right) V\right)$. We have a sequence of equalities $g=\exp \left(t_{i+1} X_{i+1}\right) \exp \left(\left(t_{0}-t_{i}\right) V\right), \forall i \geq 0$, which imply that $\exp \left(t_{i+1} X_{i+1}\right) \exp \left(-t_{i} X_{i}\right)=\exp \left(\left(t_{i}-t_{i-1}\right) V\right)$. Using the triangle inequality, we have $\left|t_{i+1}-t_{i}\right| \leq|V|_{\mathrm{bi}}\left|t_{i}-t_{i-1}\right|$. So the sequence $t_{n}$ converges to some $t^{\prime} \geq 0$. Using a suitable subsequence if necessary, we can assume that $X_{n}$ converge to some unit vector $X^{\prime}$ satisfying (4.4). Since all the geodesics $\exp \left(t X_{n}\right), t \in\left[0, t_{n}\right]$, are minimizing, the limit geodesic $\exp \left(t X^{\prime}\right), t \in\left[0, t^{\prime}\right]$ is also a minimizing geodesic from $e$ to $g \exp \left(\left(t^{\prime}-t_{0}\right) V\right)$. If $t^{\prime}>t_{0}$, then the path given by the geodesic from $e$ to $\exp \left(\left(t^{\prime}-t_{0}\right) V\right)$ and the geodesic from $\exp \left(\left(t^{\prime}-t_{0}\right) V\right)$ to $g \exp \left(\left(t^{\prime}-t_{0}\right) V\right)=\exp \left(t_{0} X\right) \exp \left(\left(t^{\prime}-t_{0}\right) V\right)$ has a length $t_{0}+|V|_{e q}\left(t^{\prime}-t_{0}\right)<t^{\prime}$, which is a contradiction. On the other hand, the condition that $\exp \left(t X_{0}\right), t \in\left[0, t_{0}\right]$, is not minimizing, implies that $t^{\prime} \neq t_{0}$. Therefore $t^{\prime} \in\left[0, t_{0}\right)$. This completes the proof of the lemma.

For any $g_{1} \neq g_{2}$, we can find $X \in \mathfrak{g}$ with $|X|_{\mathrm{bi}}=1$, such that $(X, V)$ is the Killing vector field of constant length 1 , and its flow curve, $\exp (t X) g_{1} \exp (-t V), t \in\left[0, t_{0}\right]$, generates a minimizing geodesic from $g_{1}$ to $g_{2}$ for the Randers metric $F$. Denote the local one-parameter group of diffeomorphisms generated by $(X, V)$ by $\phi_{t}$. Given $t^{\prime} \in\left[0, t_{0}\right]$, if $\phi_{t^{\prime}}$ is not a Clifford-Wolf translation, then there is some $g^{\prime} \in G$, such that the geodesic $\exp (t X) g^{\prime}, t \in\left[0, t^{\prime}\right]$, is not minimizing. Then with respect to the bi-invariant metric, the geodesic $\exp (t X), t \in\left[0, t^{\prime}\right]$, does not minimizes the distance from $e$ to $\exp \left(t^{\prime} X\right)$. By Lemma 4.2, there is $X^{\prime \prime}$ with $\left|X^{\prime \prime}\right|_{\mathrm{bi}}=1$ and $t^{\prime \prime} \in\left[0, t^{\prime}\right)$, such that $\exp \left(t^{\prime} X\right)=\exp \left(t^{\prime \prime} X^{\prime \prime}\right) \exp \left(\left(t^{\prime}-t^{\prime \prime}\right) \operatorname{Ad}\left(g_{1}\right) V\right)$. This means that the geodesic $\exp \left(t X^{\prime \prime}\right) g_{1} \exp (-t V), t \in\left[0, t^{\prime \prime}\right]$, gives a path with length $t^{\prime \prime}$. It is shorter than the geodesic $\exp (t X) g_{1} \exp (-t V), t \in\left[0, t^{\prime}\right]$, from $g_{1}$ to $\exp \left(t^{\prime} X\right) g_{1} \exp \left(-t^{\prime} V\right)$. This is a contradiction with the fact that for $t \in\left[0, t_{0}\right],(X, V)$ generates a minimizing geodesic from $g_{1}$ to $g_{2}$. Therefore $(G, F)$ is CW-homogeneous. This completes the proof of Theorem 1.1.

\section{$5 \quad$ Proof of Theorem 4.1}

\subsection{The theme of the proof}

Let $G$ be a compact connected simple Lie group with Lie algebra $\mathfrak{g}, \mathfrak{h}$ a Cartan subalgebra of $\mathfrak{g}$ with $\operatorname{dim}_{\mathbb{R}} \mathfrak{h}=\operatorname{rk}(\mathfrak{g})=n$, and $W$ the corresponding Weyl group. The bi-invariant metric on $G$ induces a $W$-invariant linear metric on $\mathfrak{h}$. The condition in Theorem 4.1, that the vector field generated by $X \in \mathfrak{g}$ is not a Killing vector field of constant length of the left invariant Randers metric $F$ on $G$ when $F$ is not Riemannian, 
is equivalent to the condition that any ellipsoid containing the $\operatorname{Ad}(G)$-orbit $X$ must be centered at 0 .

If - Id is contained in the Weyl group, the proof goes easily by applying the next lemma.

Lemma 5.1 If -Id belongs to the Weyl group of a simple compact Lie algebra $\mathfrak{g}$, then any ellipsoid containing the $\operatorname{Ad}(G)$-orbit of a non-zero element $X \in \mathfrak{g}$ must be centered at 0 .

Proof. We only need to prove that for any non-zero $X$ in a Cartan subalgebra, an ellipsoid containing its Weyl group orbit must be centered at 0 . Denote the equation of the ellipsoid $E$ containing the Weyl group orbit of $X \neq 0$ as

$$
x^{T} A x+b^{T} x+c=0 .
$$

Then the ellipsoid defined by the equation

$$
x^{T} A x-b^{T} x+c=0
$$

also contains the Weyl group orbit of $X$. So the Weyl group orbit of $X$ is contained in the subspace $b^{T} x=0$ if $b \neq 0$, or equivalently, the ellipsoid $E$ is not centered at 0 . This conflicts with the fact that the representation of the Weyl group is irreducible on the Cartan subalgebra of a simple compact $\mathfrak{g}$.

It is well known that, the Weyl groups of all simple compact Lie algebras except $A_{n}$ with $n>1, D_{n}$ with $n$ odd, and $E_{6}$, contain the endomorphism - Id on the Cartan subalgebras. For the last three cases, we need another lemma.

Let $\left\{v_{1}, \ldots, v_{n}\right\}$ be a basis of the vector space $\mathfrak{h}$ given by roots, with corresponding reflections $\rho_{1}, \ldots, \rho_{n}$ in the Weyl group. Denote the linear subspace spanned by $\rho_{i}$ as $v_{i}^{\perp}$. Then we have

Lemma 5.2 Suppose for any $i=1, \ldots, n$, we can find $n$ points on the Weyl group orbit of $X$ outside $v_{i}^{\perp}$, such that their orthogonal projections in $v_{i}^{\perp}$ form an affine basis of $v_{i}^{\perp}$. Then any ellipsoid containing the Weyl group orbit of $X$ must be centered at the origin.

Proof. Let $E$ be the ellipsoid containing the Weyl group orbit of $X$. Suppose $l$ is a line which has two intersectional points with $E$ and denote by $L$ the set of all the lines which is parallel to $l$. Define $D$ to be the set of the middle points of the intersectional points of the lines in $L$ with $E$. Then $D$ is contained in a hyperplane. Notice that the center of the ellipsoid must be contained in this hyperplane. For each $i=1, \ldots, n$, the hyperplane containing all middle points with the lines parallel to $v_{i}$ contains a set of $n$ projections from the orbit of $X$, which gives an affine basis. Thus it is identical with $v_{i}^{\perp}$. Since the $v_{i}^{\perp}$ 's have the unique common point (the origin), the center of the ellipsoid must be the origin.

The generic condition for $X$ are certain conditions for the eigenvalue multiplicities for the cases of $A_{n}$ and $D_{n}$, and more generally the isomorphic type of its isotropy group for $E_{6}$. They are invariant under Ad-actions, namely, if $X$ is generic, then each element of the Ad-orbit of $X$ is also generic. If a generic element $X$ generates a Killing 
vector field of constant length for some Randers metric $F$, then the restriction of $F$ to each Cartan subalgebra must be Riemannian, since the indicatrix ellipsoid is centered at 0 . This implies that $F$ must be Riemannian. Therefore we are left to calculate the codimension of the complement of the set of all generic elements in $\mathfrak{g}$. Next we deal with this problem case by case.

\subsection{The case of $A_{n}$ with $n>1$}

The diagonal matrices in $\mathfrak{s u}(n+1)$ with $n>0$ form a Cartan subalgebra. Any matrix in it can be identified with a vector $X=\left(a_{0}, \ldots, a_{n}\right) \in \mathbb{R}^{n+1}$, in which $\sum_{i=0}^{n} a_{i}=0$, and the eigenvalues are $a_{i} \sqrt{-1}, i=0, \ldots, n$. The weyl group is the full permutation group $S_{n+1}$ for all the entries of $X$.

Take $v_{i}=(1,0, \ldots, 0,-1,0, \ldots, 0)$ with -1 in the $i$ th-entry. The reflection $\rho_{i}$ is the permutation which interchanges $a_{0}$ and $a_{i}$, and fixes all the other entries.

Let $X$ be a vector with the first entry equal to $a$, the last entry equal to $b$ such that $b<a$ and suppose all the other entries $c_{i}$ lies in $\mathbb{R} \backslash\{a, b\}, i=1, \ldots, n-1$. Then using the action of $\rho_{i}, i=0,1, \ldots, n$, we get the following $n$ points in the Weyl group orbit of $X$ :

$$
\begin{aligned}
X_{0} & =\left(a, b, c_{1}, \ldots, c_{n-1}\right), \\
X_{i} & =\left(a, c_{i}, c_{1}, \ldots, c_{i-1}, b, c_{i+1}, \ldots, c_{n-1}\right),
\end{aligned}
$$

for $i=1, \ldots, n-1$. It is easy to see that neither of the above points is contained in $v_{1}^{\perp}$. To see that their orthogonal projections form an affine basis for $v_{1}^{\perp}$, we only need to notice the fact that the set of vectors

$$
\operatorname{pr}\left(X_{i}\right)-\operatorname{pr}\left(X_{0}\right)=\left(\left(c_{i}-b\right) / 2,0, \ldots, 0, c_{i}-b, 0, \ldots, 0\right), \forall i=1, \ldots, n-1,
$$

are linearly independent, which is obvious. The same technique can also be applied to other $v_{i}^{\perp}$ with $i>1$. Therefore the condition of Lemma $[5.2$ is satisfied. Hence the ellipsoid containing this $X$ must be centered at 0 . Thus the left-invariant Randers metric with Killing vector fields of constant length given by this $X$ must be Riemannian on each Cartan subalgebra. Therefore $F$ is Riemannian.

Any matrix in $\mathfrak{s u}(n+1)$ have $n+1$ imaginary eigenvalues, with their multiplicities denoted as $\left\{n_{1}, \ldots, n_{m}\right\}, \sum_{i=1}^{m} n_{i}=n+1$. The generic condition discussed above is in fact that there are two 1's among all $n_{i}$ 's. The matrix space $\mathfrak{s u}(n+1)$ can be naturally stratified as a finite union of subvarieties with respect to the multiplicities. Notice that the isotropy group for the $\mathrm{A} d(S U(n))$-action at $X \in \mathfrak{s u}(n+1)$ with eigenvalue multiplicities $\left\{n_{1}, \ldots, n_{m}\right\}$ is $S\left(U\left(n_{1}\right) \times \cdots \times U\left(n_{m}\right)\right)$. So the set of the matrices with the same eigenvalue multiplicities $\left\{n_{1}, \ldots, n_{m}\right\}$ is a subvariety with codimension

$$
\sum_{i=1}^{m} n_{i}^{2}-m=\sum_{i=1}^{m}\left(n_{i}+1\right)\left(n_{i}-1\right) \geq n+m-2 l,
$$

in which $l$ is the number of 1 's among the $n_{i}$ 's. Obviously, when $m \geq 3$ and $l \leq 1$, the codimension is $n+m-2 l \geq n+1$, and when $m=2$ and $l \leq 1$, it is also easy to see that the inequality in (5.8) is restrict. Thus each stratified subset in the complement of the set of the generic elements has a codimension at least $n+1$, and so does their union. This completes the proof of Theorem 4.1 for the case of $A_{n}$. 


\subsection{The case of $D_{n}$ with odd $n>2$}

In a Cartan subalgebra of $\mathfrak{s o}(2 n)$ with $n>2$, the matrix can be identified with a vector $X=\left(a_{1}, \ldots, a_{n}\right) \in \mathbb{R}^{n}$, such that the eigenvalues of the matrix are $\pm a_{i} \sqrt{-1}$ for $i=1, \ldots, n$. The Weyl group actions permute the entries and change even numbers of signs of the entries arbitrarily. The eigenvalue multiplicities for a matrix in $\mathfrak{s o}(2 n)$ can be denoted as $\left\{n_{0}, n_{1}, \ldots, n_{m}\right\}$, where $n_{0}$ is the even multiplicity of the eigenvalue 0 , and the $n_{i}$ 's are eigenvalue multiplicities for positive multiples of $\sqrt{-1}$. Then we have $n_{0}+2 \sum_{i=1}^{m} n_{i}=2 n$. Suppose the eigenvalue multiplicities $X \in \mathbb{R}^{n}$ satisfies the conditions that $m \geq 2$ and there is an $i>0$ such that $n_{i}=1$. After a suitable change of the order and adjustment of the signs, we can suppose that $X$ can be represented as $\left(a, b, c_{1}, \ldots, c_{n}\right)$ such that $a$ and $b$ are non-zero, and $a$ has different absolute value from the others. If there is a $c_{i}$ such that $c_{i}=b$, then we change the sign for that $c_{i}$ and $a$ simultaneously using a Weyl group element. Therefore we can assume further that $b \neq c_{i}$, for $i=1, \ldots, n-2$. Let $v_{i}=(1,0, \ldots,-1,0, \ldots, 0)$ be the root with $\mathrm{a}-1$ in the $(i+1)$-th entry, and denote $v_{0}=(1,1,0, \ldots, 0)$. One can find the following $n$ points from the Weyl group orbit of $X$ outside $v_{1}^{\perp}:\left(a, b, c_{1}, \ldots, c_{n-2}\right)$, $\left(a, c_{i}, c_{1}, \ldots, c_{i-1}, b, c_{i+1}, \ldots, c_{n-2}\right)$ with $i=1, \ldots, n-2$, and $\left(-a,-b, c_{1}, \ldots, c_{n-2}\right)$. Neither of the $n$ points is contained in $v_{1}^{\perp}$, and their orthogonal projections in $v_{1}^{\perp}$ form an affine basis. This argument can also applied to $v_{0}$ and the other $v_{i}$ 's. Thus $X$ is a generic vector in the sense of Lemma 5.2,

In this case the generic condition for $X$ can be stated as follows: the eigenvalue multiplicities $\left\{n_{0}, \ldots, n_{m}\right\}$ of $X$ satisfy the condition that $m \geq 2$, and there is a $n_{i}=1$ for some $i>0$. The isotropy group of the $\operatorname{Ad}(\mathrm{SO}(2 n))$-action at $X$ with eigenvalue multiplicities $\left(n_{0}, \ldots, n_{m}\right)$ is isomorphic to $\mathrm{SO}\left(n_{0}\right) \times \mathrm{U}\left(n_{1}\right) \times \mathrm{U}\left(n_{m}\right)$. Therefore the subvariety of the elements $X$ whose eigenvalue multiplicities are all the same, has codimension $n_{0}\left(n_{0}-1\right) / 2+\sum_{i=1}^{m} n_{i}^{2}-m$. If $m=1$ and $n_{1}=1$, then the codimension equals $(n-1)(2 n-3)>n+1$ when $n>2$. If for all $i>0$ we have $n_{i}>1$, then the codimension is

$$
\begin{aligned}
\frac{n_{0}\left(n_{0}-1\right)}{2}+\sum_{i=1}^{m} n_{i}^{2}-m & =\frac{n_{0}\left(n_{0}-1\right)}{2}+\sum_{i=1}^{m}\left(n_{i}-1\right)\left(n_{i}+1\right) \\
& \geq n_{0}-1+\sum_{i=1}^{m} n_{i}+m \\
& =n+\left(m+\frac{n_{0}}{2}-1\right) \geq n
\end{aligned}
$$

in which the equality holds only when $n_{0} / 2+m=1$. However, one can easily check that the equality can not hold. So in the complement of the set of the generic elements, any stratified subvariety of the fixed type of eigenvalue multiplicities has a codimension at least $n+1$. This completes the proof of Theorem 4.1 for the case of $D_{n}$.

\subsection{The case of $E_{6}$}

Now we consider the last case of $E_{6}$, which is also the most difficult one. The Cartan subalgebra of $E_{6}$ can be identified with $\mathbb{R}^{6}$. The root system consists of $\alpha=$ $( \pm 1, \pm 1,0,0,0,0)$ together with all permutations of $\alpha$ keeping the last entry 0 fixed, and 
all the vectors $( \pm 1 / 2, \pm 1 / 2, \pm 1 / 2, \pm 1 / 2, \pm 1 / 2, \pm \sqrt{3} / 2)$ with odd positive signs. It is easy to observe that the set $\Pi$ of roots which are perpendicular to $(1 / 2, \ldots, 1 / 2,-\sqrt{3} / 2)$ consists of the permutations of $(1,-1,0,0,0,0)$, together with the permutations of the vectors $\pm(1 / 2, \ldots, 1 / 2,-1 / 2, \sqrt{3} / 2)$, both keeping the last entry fixed. It is also easily checked that the set set $\Pi$ forms the root system of $A_{5}$. We can use an orthogonal automorphism of $\mathbb{R}^{6}$ to map them to the standard root system of $A_{5}$, i.e., all permutations of $(1,-1,0,0,0,0)$. More precisely, the orthogonal automorphism keeps all permutations of $(1,-1,0,0,0,0)$ fixing the last entry 0 , and maps $(-1 / 2, \ldots,-1 / 2,1 / 2,-\sqrt{3} / 2)$ to $(0, \ldots, 0,1,-1)$. We can also assume that it maps the roots $\pm(1 / 2,1 / 2,1 / 2,1 / 2,1 / 2,-\sqrt{3} / 2)$ to $\pm\left(3^{-1 / 2}, \ldots, 3^{-1 / 2}\right)$, respectively. It is not hard to check that it maps the remaining forty roots to $\pm\left(\left(3^{-1 / 2} \pm 1\right) / 2, \ldots,\left(3^{-1 / 2} \pm 1\right) / 2\right)$ with three positive signs and three negative signs among the six entries. To summarize, we have the following lemma.

Lemma 5.3 The root system of $E_{6}$ can be represented as the union of the standard root system of $A_{5} \times A_{1}$, i.e., all permutations of $(1,-1,0, \ldots, 0)$ and $\pm\left(3^{-1 / 2}, \ldots, 3^{-1 / 2}\right)$, and all the vectors $\pm\left(\left(3^{-1 / 2} \pm 1\right) / 2, \ldots,\left(3^{-1 / 2} \pm 1\right) / 2\right)$ with three positive signs and three negative signs among the six entries.

For any vector $X \in \mathbb{R}^{6}$, or the Cartan subalgebra of $E_{6}$, the multiplicities of its different entries can be denoted as a set of positive integers $\left\{n_{1}, \ldots, n_{m}\right\}$, with $\sum_{i=1}^{m} n_{m}=6$. For simplicity, we will call it the multiplicity type of $X$. Suppose $X$ is a vector in the Cartan subalgebra whose all entries sum to 0, i.e., a vector in the Cartan subalgebra of $A_{5}$. From Subsection 5.2. we see that if the multiplicity type of $X$ contains two 1's, then any ellipsoid in the Cartan subalgebra of $A_{5}$ containing the $A_{5}$-Weyl group orbit of $X$ is centered at 0 . In fact it is true for more generic elements.

Lemma 5.4 If $X$ has three different entries and the sum of all its entries is equal to 0 , then any ellipsoid in the Cartan subalgebra of $A_{5}$ which contains the $A_{5}$-Weyl group orbit of $X$ must be centered at 0 .

Proof. We only need to consider the case with multiplicity types $\{1,2,3\}$ and $\{2,2,2\}$ and repeat the arguments in Subsection 5.2 .

Consider $X=(a, b, b, c, c, c)$ where $a, b$ and $c$ are three different numbers with $a+2 b+3 c=0$. Let the roots $v_{i}=(1,0, \ldots, 0,-1,0, \ldots, 0)$ with a -1 in the $(i+1)$-th entry, $i=1, \ldots, 5$. For $v_{1}=(1,-1,0, \ldots, 0)$, the $A_{5}$-Weyl group orbit of $X$ contains the points $(a, b, b, c, c, c),(c, b, b, a, c, c),(c, b, b, c, a, c),(c, b, b, c, c, a)$ and $(a, b, c, b, c, c)$. They are vectors outside $v_{1}^{\perp}$ and their orthogonal projections form an affine basis for $v_{1}^{\perp}$. For other $v_{i}$ 's, the arguments are similar. By Lemma 5.2, any ellipsoid containing the $A_{5}$-Weyl group orbit of $X$ is centered at 0 .

Consider $X=(a, a, b, b, c, c)$ where $a, b$ and $c$ are three different numbers with $a+$ $b+c=0$. Then for $v_{1}$, the $A_{5}$-Weyl group orbit of $X$ contains the points $(a, b, a, b, c, c)$, $(a, b, b, a, c, c),(a, b, a, c, b, c),(a, b, a, c, c, b)$ and $(a, c, a, b, c, b)$. They are vectors outside $v_{1}^{\perp}$ and their projections form an affine basis for $v_{1}^{\perp}$. Similar arguments also apply to other $v_{i}$ 's. This completes the proof of the lemma.

Finally, we prove that any ellipsoid containing the $E_{6}$-Weyl group orbit of $X$ must be centered at 0 , provided that $X$ has three different entries. 
First we consider the case that the sum of all entries of $X$ is not 0 . Let $E$ be any ellipsoid containing the $A_{5} \times A_{1}$-Weyl group orbit of $X$. Notice that the Cartan subalgebra of the $A_{1}$ factor is generated by $v_{0}=\left(3^{-1 / 2}, \ldots, 3^{-1 / 2}\right)$, and the Cartan subalgebra of $A_{5}$ is the orthogonal complement $v_{0}^{\perp}$. The $A_{5} \times A_{1}$-Weyl group orbit of $X$ has no intersection with $v_{0}^{\perp}$, and Lemma 5.4 implies that their orthogonal projection to $v_{0}^{\perp}$ contains an affine basis for $v_{0}^{\perp}$. So the middle points of the intersection between $E$ and the lines parallel to $v_{0}$ are contained in $v_{0}^{\perp}$. Similarly, the centers of the intersection ellipsoid between $E$ and the hyperplanes parallel to $v_{0}^{\perp}$ is contained in $\mathbb{R} v_{0}$. Since the center of $E$ must be contained in both $v_{0}^{\perp}$ and $\mathbb{R} v_{0}$, it must be 0 .

Next we consider the case that the sum of all entries of $X$ is 0 . Let $E$ be any ellipsoid containing the $E_{6}$-Weyl group orbit of $X$. Then Lemma 5.4 implies that the intersection of $E$ with $v_{0}^{\perp}$ is an ellipsoid containing the $A_{5}$-Weyl group orbit of $X$, which is centered at 0 . On the other hand, any element $\rho$ in the $E_{6}$-Weyl group maps $v_{0}^{\perp}$ to another subspace. Thus the intersection between $E$ and $\rho\left(v_{0}^{\perp}\right)$ is also an ellipsoid containing the $\rho$-images of the $A_{5}$-Weyl group orbit of $X$, which is also centered at 0 . Therefore the center of $E$ must be 0 , since it is contained in both the lines generated by $v_{0}$ and $\rho\left(v_{0}\right)$ which intersect at 0 . This proves the assertion.

For non-zero vector $X$ with other multiplicity types, namely, $\{6\},\{5,1\},\{4,2\}$ and $\{3,3\}$, if we can find a vector $X^{\prime}$ in its $E_{6}$-Weyl group orbit with three different entries, the same statement is still true. If we assume further that any vector in its Weyl group orbit does not have three different entries, then additional linear relations will be required between the entries of $X$. So up to nonzero constant scalars, we can only find finite $X$ 's in the Cartan subalgebra $\mathfrak{h}$, even if there does exist such $X$. The centralizer of such a $X$ is the Lie algebra of the isotropy group for the $\operatorname{Ad}(G)$-action. It contains $\mathfrak{h}$ and is obviously not abelian. Hence its dimension is at least $n+2$. So the union of all the $\operatorname{Ad}(G)$-orbits of the generic elements in the above sense has a codimension at least $n+1$ ( the dimension of the centralizers minus the one dimension for scalar changes). Thus the assertion of Theorem 4.1 holds for $E_{6}$. This completes the proof of Theorem 4.1 .

Acknowledgements. We would like to thank Dr. Libing Huang and Dr. Zhiguang $\mathrm{Hu}$ for useful discussions. This work was finished during the second author's visit to the Chern institute of Mathematics. He would like to express his deep gratitude to the members of the institute for their hospitality.

\section{References}

[AW76] R. Azencott, E. Wilson, Homogeneous manifolds with negative curvature I, Trans. Amer. Math. Soc., 215 (1976), 323-362.

[BCS00] D. Bao, S. S. Chern, Z. Shen, An Introduction to Riemann-Finsler Geometry, Springer-Verlag, New York, 2000.

[BN08-1] V. N. Berestovskii, Yu. G. Nikonorov, Killing vector fields of constant length on locally symmetric Riemannian manifolds, Transformation Groups, 13 (2008), $25 \mathrm{C} 45$. 
[BN08-2] V. N. Berestovskii, Yu.G. Nikonorov, On $\delta$-homogeneous Riemannian manifolds, Diff. Geom. Appl., 26 (2008), 514C535.

[BN09] V. N. Berestovskii, Yu.G. Nikonorov, Clifford-Wolf homogeneous Riemannian manifolds, Jour. Differ. Geom., 82 (2009), 467-500.

[CS05] S. S. Chern, Z. Shen, Riemann-Finsler Geometry, World Scientific Publishers, 2004.

[DH02] S. Deng and Z. Hou, The group of isometries of a Finsler space, Pacific J. Math, 207 (2002), 149-157.

[DMW86] I. Dotti-Miatello, R. Miatello and J. A. Wolf, Bounded isometries and homogeneous Riemannian quotient manifolds, Geom. Dedicata, 21 (1986), 21C27.

[DE12] S. Deng, Clifford-Wolf translations of Finsler spaces of negative flag curvature, preprint.

[DM12] S. Deng and M. Xu, Clifford-Wolf translations of Finsler spaces, to appear in Forum Math., [Math.DG] arXiv:1201.3714v1.

[DR83] M. J. Druetta, Clifford translations in manifolds without focal points, Geom. Dedicata, 14 (1983), 95C103.

[FR63] H. Freudenthal, Clifford-Wolf-isometrien symmetrischer raüme, Math. Ann., 150 (1963), 136-149.

[HE74] E. Heintze, On homogeneous manifolds of negative curvature, Math. Ann., 211 (1974), 23-34.

[OT76] T. Ochiai and T. Takahashi, The group of isometries of a left invariant metric on a Lie group, Math. Ann., 223 (1976), 91-96.

[OZ69] V. Ozols, Critical points of the displacement function of an isometry, J. Differential Geometry, 3 (1969), 411C432.

[OZ74] V. Ozols, Clifford translations of symmetric spaces, Proc. Amer. Math. Soc., 44 (1974), 169C175.

[SH01] Z. Shen, Differential Geometry of Sprays and Finsler Spaces, Kluwer, Dordrent, 2001.

[WO60] J. A. Wolf, Sur la classification des varietes riemanniennes homogenes a courbure constante, C. R. Math. Acad. Sci. Paris, 250 (1960), 3443-3445.

[WO62] J. A. Wolf, Locally symmetric homogeneous spaces, Commentarii Mathematici Helvetici, 37 (1962/63), 65-101.

[WO64] J. A. Wolf, Homogeneity and bounded isometries in manifolds of negative curvature, Illinois J. Math., 8 (1964), 14-18. 